PROCEEDINGS OF THE

AMERICAN MATHEMATICAL SOCIETY

Volume 49, Number 2, June 1975

\title{
LEMMA ON MEASURABLE CARDINALS
}

\author{
WILLIAM G. F LEISSNER
}

ABSTRACT. An ordinal is moved by only finitely many measurable cardinals.

We inductively define a class $M$, analogously to $L$, Gödel's class of constructible sets. Let $M_{0}=\varnothing ; M_{\lambda}=\bigcup\left\{M_{a}: \alpha<\lambda\right\}$; and let $M_{\alpha+1}$ be the collection of subsets of $M_{a}$ definable using parameters by some formula of the infinitary language $L_{\omega_{1} \omega_{1}}$ (instead of $L_{\omega \omega}$, the usual finitary language). Let $M=\bigcup\left\{M_{\alpha}: \alpha \in \mathrm{OR}\right\}$; Chang [1] called this class $C_{\omega_{1}}$. Kunen [2] used the lemma below to show that if there are $\omega_{1}$ measurable cardinals, then the axiom of choice is false in M. Kunen's proof used the machinery of iterated ultrapowers. The present proof can be read by anyone familiar with the fundamental paper of Scott [3].

A free $\kappa$-complete ultrafilter $U$ on $\kappa$ induces an elementary embedding $j$ of $V$ into the transitive realization $N$ of the ultrapower $V^{\kappa} / U$. We say $\kappa$ moves $\delta$ when there is a $U$ on $\kappa$ so that $j(\delta) \neq \delta$.

Lemma. An ordinal is moved by only finitely many measurable cardinals.

Proof. Suppose $j(\delta) \neq \delta$. Then there is a maximal interval of ordinals moved by $j$ containing $\delta$. It is straightforward to show that this interval is of the form $[\alpha, \beta)$ where $\operatorname{cf}(\alpha)=\kappa$, and $\beta=\sup ^{n}(\gamma)$ for $\gamma \in[\alpha, \beta)$.

Claim 1. If $\kappa_{0}<\kappa_{1}$ then $j_{1}\left(j_{0}(\gamma)\right)=j_{0}\left(j_{1}(\gamma)\right)$.

Informally, the idea is that $j_{1}\left(j_{0}(\gamma)\right)$ is $N_{1}$ 's notion of the $j_{0}$ process applied to $j_{1}(\gamma)\left(N_{1}\right.$ 's notion of $\left.\gamma\right)$, which is $j_{0}\left(j_{1}(\gamma)\right)$ because $N_{1}$ 's notion is correct.

Let $\phi_{i}\left(x, y, U_{i}\right)$ be a formula of the language of $Z F$, obtained in the familiar manner, such that for all $x, y, \phi_{i}\left(x, y, U_{i}\right)$ iff $j_{i}(x)=y(i=0,1)$.

Then for every $\gamma \in \mathrm{OR}, \phi_{0}\left(\gamma, j_{0}(\gamma), U_{0}\right)$. Clearly

$$
\begin{aligned}
\phi_{0}\left(\gamma, j_{0}(\gamma), U_{0}\right) & \Leftrightarrow \phi_{0}^{j_{1}^{\prime \prime} V}\left(j_{1}(\gamma), j_{1}\left(j_{0}(\gamma)\right), j_{1}\left(U_{0}\right)\right) \\
& \Leftrightarrow \phi_{0}^{N_{1}}\left(j_{1}(\gamma), j_{1}\left(j_{0}(\gamma)\right), U_{0}\right) \Leftrightarrow \phi_{0}\left(j_{1}(\gamma), j_{1}\left(j_{0}(\gamma)\right), U_{0}\right) .
\end{aligned}
$$

Received by the editors April 16, 1974 and, in revised form, September 6, 1974. AMS (MOS) subject classifications (1970). Primary 02K 35 .

Key words and phrases. Measurable cardinal. 
Since $j_{0}\left(j_{1}(\gamma)\right)$ is the unique $y$ such that $\phi_{0}\left(j_{1}(\gamma), y, U_{0}\right)$, we get from $\phi_{0}\left(j_{1}(\gamma), j_{1}\left(j_{0}(\gamma)\right), U_{0}\right)$

$$
j_{1}\left(j_{0}(\gamma)\right)=j_{0}\left(j_{1}(\gamma)\right)
$$

Claim 2. If $\left[\alpha_{0}, \beta_{0}\right)$ and $\left[\alpha_{1}, \beta_{1}\right)$ intersect, they are nested. Suppose $a_{0}<a_{1}$. Let $a_{0}<\gamma<a_{1}, j_{1}(\gamma)=\gamma$. Then $j_{1}\left(j_{0}^{n}(\gamma)\right)=j_{0}^{n}(\gamma)$, so no $j_{0}^{n}(\gamma) \epsilon$ $\left[\alpha_{1}, \beta_{1}\right)$. Then either $\beta_{0}=\sup j^{n}(\gamma)<\alpha_{1}$ or there is $n$ so that $j_{0}^{n}(\gamma)<\alpha_{1}<$ $\beta_{1}<j_{0}^{n+1}(\gamma)$.

Let $\delta,\left\{\kappa_{i}: i \in \omega\right\}$ be a counterexample to the Lemma. Since cf $\left(\alpha_{i}\right) \neq$ cf $\left(\alpha_{j}\right)$ there is an infinite ascending chain $\left\{\alpha_{i}: i \in \omega\right\}$ of ordinals. By Claim 2 , there is an infinite descending chain of ordinals. Contradiction.

\section{REFERENCES}

1. C. C. Chang, Sets constructible using $L_{\kappa K}$, Proc. Sympos. Pure Math., vol. 13, part I, Amer. Math. Soc. Providence, R. I., 1971, pp. 1-8. MR 43 \#6077.

2. K. Kunen, $A$ model for the negation of the axiom of choice, Proc. Cambridge Summer School in Logic, 1971.

3. D. Scott, Measurable cardinals and constructible sets, Bull. Acad. Polon. Sci. Sér. Sci Math. Astronom. Phys. 9 (1961), 521-524. MR 26 \#1263.

DEPARTMENT OF MATHEMATICS, UNIVERSITY OF WISCONSIN, MADISON, WISCONSIN 53706

Current address: Department of Mathematics, McGill University, Montreal, Quebec, Canada $\mathrm{H} 3 \mathrm{C} 3 \mathrm{G} 1$ 